\title{
A Randomized Approach to Probabilistic Footprint Estimation of a Space Debris Uncontrolled Reentry
}

\author{
Alessandro Falsone, Student Member, IEEE and Maria Prandini, Senior Member, IEEE
}

\begin{abstract}
This paper studies the problem of characterizing the region of the airspace that will be occupied by a space debris during an uncontrolled reentry (footprint), with the final goal of supporting the air traffic controllers in their task of guiding aircraft safely from their origin to their destination. Given the various sources of uncertainty affecting the debris dynamics, the reentry process is characterized probabilistically, and the problem of determining the footprint is formulated in terms of a chance-constrained optimization program, which is solved via a simulation-based method. When observations of the debris initial position and radar measurements of the aircraft prior to the reentry event are available, nonlinear filtering techniques can be adopted and the posterior probability distribution of the debris position as well as of the wind field affecting the reentry process can be integrated in the chance-constraint formulation so as to obtain an enhanced estimate of the footprint. Simulation results show the efficacy of the approach.
\end{abstract}

Index Terms-Randomized algorithms; nonlinear filtering; air traffic control; uncontrolled debris reentry.

\section{INTRODUCTION}

Since the beginning of the space era the number of satellites grew significantly and so did the number of them which ceased their operations and now are orbiting around Earth without the possibility to be controlled from ground stations. Together with upper stage rocket bodies, and fragments generated by collisions with meteoroids or other artificial satellites, they constitute the so-called space debris population. When its orbit decays, mainly due to atmospheric friction, a debris can reenter the atmosphere. If it can be manoeuvred, then, high density fly zones are avoided and the debris is typically driven to strike the ground in the ocean or over an uninhabited area. In the case of uncontrolled reentry, instead, the debris cannot be manoeuvred and, hence, poses risk to public safety, striking people and properties on the ground as well as aircraft in the air. Interestingly, notwithstanding the fact that aircraft vulnerability is higher compared to that of people on the ground (in the case of an aircraft flying at a speed of 700 kilometers per hour even an impact with a small debris, hypothetically at rest in the air, can cause a severe damage, [1]), the aviation risk has started being considered only lately and in a few contributions (see e.g. [2]-[4]), the main concern being the possible impact in terms of casualties on the population on the ground (see [1], [5]-[8] to name a few).

Up today, accurate and reliable models to describe uncontrolled space debris reentry are not available, due to the

Alessandro Falsone and Maria Prandini are with the Dipartimento di Elettronica, Informazione e Bioingegneria, Politecnico di Milano, Italy \{alessandro.falsone, maria.prandini\}@polimi.it

* This work is partially supported by the European Commission under the project UnCoVerCPS with grant number 643921. complexity of the phenomenon. When a satellite ceases its operation, it typically continues to orbit around the Earth until the atmospheric drag reduces its speed making it lower its major semi-axis. Typical speed of an orbiting satellite is about $7 \mathrm{~km} / \mathrm{s}$ and, as it enters the Earth atmosphere, it encounters a steadily increasing aerodynamic load that causes a sequence of failures, thus generating multiple fragments, [3]. Though this fragmentation is far from instantaneous, we refer to it as the breakup instant, meaning the instant when the reentering body experiences a major breakup (i.e. the first massive breakup). In [6], [7] it is shown that all fragments evolve with a ballistic trajectory, only subject to gravity and aerodynamic forces. Observations have shown that the major breakup event happens at an altitude of about 78 $\mathrm{km}$ [3] with an uncertainty range from $\pm 10 \mathrm{~km}$ to $\pm 20 \mathrm{~km}$, [6]-[8]. So, fragments generated from this first breakup are distributed over a range of altitudes, thus increasing the spread of debris footprint along the direction of motion. Following [3], which suggests that the spread of debris footprint caused by breakup altitude uncertainty can be reduced if the object is observed during the major breakup, we focus only on fragment dispersion after the breakup phase.

Besides the complex nature of the breakup process, even post-breakup trajectories of fragments are subject to strong uncertainties. In particular, the aerodynamic drag acting on a fragment depends on the ballistic coefficient, which accounts for the shape and mass of the object and is uncertain, and on the atmospheric density, that is affected by modeling errors, [1], [9]. Local wind is pointed out as a source of uncertainty in [3], [10], due to its effect on the debris trajectories after the breakup process, when the debris enters the low atmosphere. The horizontal component of the velocity vector is in fact typically dominated by the wind that can cause a cross-track dispersion of the debris, [3], the impact being higher on those fragments with a lower ballistic coefficient, [10].

All this motivated the adoption of a probabilistic approach in previous works in the literature (see e.g. [4], [6], [7]), and in this paper as well.

Our goal here is to propose a method for determining the footprint of a debris fragment, i.e., the minimum size 4D (space cross time) region of the airspace where all fragment trajectories except a set of predefined probability $\varepsilon \in(0,1)$ can be confined. More specifically, we introduce a simulationbased approach where the problem of the $\varepsilon$-footprint computation is formulated as a chance-constrained optimization problem and then solved through a randomized method, which reduces to generating a certain number of realizations of the reentry trajectory and imposing that these realizations belong to the footprint. The availability of measurements of the debris 
position can be used to decrease the size of the footprint, through nonlinear filtering techniques and, in particular, the unscented Kalman filter [11]. A further improvement of the footprint estimate can be obtained by exploiting the radar measurements of the aircraft positions during normal operations. Given the strong correlation in time of the wind field, the particle filtering technique proposed in [12] can be effective in reducing the uncertainty on the wind acting on the debris trajectory. Numerical examples are presented throughout the paper to assess the performance of the proposed approach.

This paper significantly extends our preliminary work in [4] by introducing footprint refinement based on available measurements, combining unscented Kalman filtering and sequential particle filtering to this purpose. It can be seen as a first step for the introduction of automatic tools that, as soon as a debris enters the atmosphere, provide an estimate of the airspace area that will be occupied by the reentering debris. Air traffic controllers - who are in charge of monitoring air traffic and ensuring safe flights, [13], [14] - could then decide the best action in terms of re-routing of the aircraft involved so as to limit service disruption and avoid fatalities in the air.

The rest of the paper is organized as follows. In Section II, we characterize the $4 \mathrm{D} \varepsilon$-footprint of a debris fragment and propose a simulation-based method for its computation, using the model for a single-fragment reentry trajectory simulation in [15] (Section III). The state of the art analytic approach for estimating the footprint in [15] is compared with our approach in Section IV. Footprint refinement via nonlinear filtering techniques is addressed in Section $\mathrm{V}$, considering first the measurements of the debris (Section V-A) and then those of the aircraft (Section V-B). The algorithm for enhanced footprint estimation is given in Section V-C. A simulation example is presented in Section VI. Finally, in Section VII, we draw some conclusions and suggest directions of future research.

\section{Probabilistic Footprint Estimation}

The footprint of a debris fragment is the 4D (space cross time) region of the airspace that will be occupied by the fragment during its uncontrolled reentry, from the instant it originates due to debris breakup till it reaches the Earth surface. Given the different sources of uncertainty that affect the fragment trajectory and that make it stochastic, we shall refer to the notion of $\varepsilon$-footprint, which is the minimum size $4 \mathrm{D}$ region that contains all fragment trajectories except for a set of predefined probability $\varepsilon \in(0,1)$.

In this section, we present a randomized approach to estimate the $\varepsilon$-footprint of a debris fragment based on multiple simulations of its reentry trajectory. We shall first address the estimate of the $3 \mathrm{D} \varepsilon$-footprint associated with a certain time instant $t$, and then extend the approach to the 4D case. In both cases, ellipsoidal sets are used to describe the footprint. This can be viewed as an extension to a stochastic setting of reach set computations based on ellipses [16], [17]. Indeed, an $\varepsilon$-footprint can be reinterpreted as a probabilistic reach set, i.e., the set of states that a stochastic system can reach for all its input realizations except for a set of probability smaller than or equal to $\varepsilon$. As $\varepsilon$ approaches 0 , the more standard notion of reach set, which comprises all reachable states, is recovered. In the literature on reachability, various methods have been developed for reach sets computations of various classes of systems, see e.g. [16]-[24] to name a few. In particular, effective computational tools have been developed for non-stochastic systems. Among the different descriptions adopted in these tools, ellipses present the advantage of being a quite compact representation.

The debris dispersion at a certain time instant $t$ is described via the ellipsoidal set $\mathcal{E}_{\varepsilon}(A, \boldsymbol{c})=\left\{\boldsymbol{x} \in \mathbb{R}^{3}\right.$ : $\left.(\boldsymbol{x}-\boldsymbol{c})^{\top} A(\boldsymbol{x}-\boldsymbol{c}) \leq 1\right\}$, which is finitely parameterized through a vector $c$ and a positive definite symmetric matrix $A$ representing the center and shape of the ellipse respectively. Let $\boldsymbol{x}_{\delta}(t)$ denote the position of the debris at time $t$, when the uncertainty affecting the debris reentry trajectory is $\delta$. The uncertainty vector $\delta$ can account for various sources of uncertainties (parameters, initial condition, disturbances) as discussed in the introduction, and takes values in some set $\Delta$ according to a probability distribution $\mathcal{P}$ (see [6], [7] for sensible choices for $\mathcal{P}$ ).

The problem of computing the ellipse $\mathcal{E}_{\varepsilon}(A, \boldsymbol{c})$ with minimum volume that contains all possible debris trajectories except for a set of probability at most $\varepsilon$ can then be formalized as follows:

$$
\begin{array}{cl}
\min _{A, \boldsymbol{c}} \quad & \log \operatorname{det} A^{-1} \\
\text { subject to: } & A=A^{\top} \succ 0 \\
& \mathcal{P}\left\{\delta \in \Delta: \boldsymbol{x}_{\delta}(t) \in \mathcal{E}_{\varepsilon}(A, \boldsymbol{c})\right\} \geq 1-\varepsilon,
\end{array}
$$

where the cost to be minimized is convex as a function of the optimization variables, [25].

If we let $\left(A^{\star}, c^{\star}\right)$ be the solution to (1), then $\mathcal{E}_{\varepsilon}^{\star}=\left\{\boldsymbol{x} \in \mathbb{R}^{3}\right.$ : $\left.\left(\boldsymbol{x}-\boldsymbol{c}^{\star}\right)^{\top} A^{\star}\left(\boldsymbol{x}-\boldsymbol{c}^{\star}\right) \leq 1\right\}$ is the minimum volume ellipsoid that represents the $3 \mathrm{D}$ footprint associated with the violation parameter $\varepsilon$.

Chance-constrained optimization problems like (1) are known to be difficult to solve, [26], [27], except for specific cases like when the involved probability distribution is Gaussian. We next show how to approximately solve problem (1) via a randomized method, called the scenario approach (see [28] for a tutorial presentation), which reduces chance-constrained optimization to optimization with non probabilistic constraints by extracting a finite number $N$ of realizations of the uncertainty vector $\delta$ and replacing the constraint in probability with the $N$ constraints associated with the extracted $\delta$ 's scenarios. Guarantees on the chance-constraint feasibility of the obtained solution can be provided when the resulting scenario problem is convex and $N$ is appropriately chosen. Following [29], a fraction $k$ of the $N$ extracted scenarios can be discarded to reduce the footprint volume, while retaining chance-constraint feasibility. The scenario program to be solved is given by

$$
\begin{array}{cl}
\min _{A, \boldsymbol{c}} \quad & \log \operatorname{det} A^{-1} \\
\text { subject to: } & A=A^{\top} \succ 0 \\
& \boldsymbol{x}_{\delta^{(i)}}(t) \in \mathcal{E}_{\varepsilon}(A, \boldsymbol{c}), i \in\{1,2, \ldots, N\} \backslash I_{r},
\end{array}
$$

where $\delta^{(i)}, i=1, \ldots, N$ are extracted independently from $\Delta$ according to the distribution $\mathcal{P}$, and $I_{r}=\left\{i_{1}, \ldots, i_{k}\right\} \subset$ 
$\{1,2, \ldots, N\}$ is the set of indices of the uncertainty instances that are removed so as to improve the cost (i.e. reduce the volume of the ellipse). Note that, (2) is a convex optimization problem with a finite number of constraints, which can be solved by computing first the convex hull of all data points $\boldsymbol{x}_{\delta^{(i)}}(t), i \in\{1,2, \ldots, N\} \backslash I_{r}$, and then applying Khachiyan's algorithm to find the minimum volume ellipsoid containing the convex hull, [30], [31]. The feasibility of the scenario solution for the original chance-constrained optimization problem (1) is guaranteed by the following theorem, which derives immediately from [29].

Theorem 1. Select a confidence parameter $\eta \in(0,1)$ and an empirical violation parameter $\alpha \in[0, \varepsilon)$. If $N$ satisfies

$$
\left(\begin{array}{c}
\lfloor\alpha N\rfloor+d \\
\lfloor N\rfloor
\end{array}\right) \sum_{i=0}^{\lfloor\alpha N\rfloor+d}\left(\begin{array}{c}
N \\
i
\end{array}\right) \varepsilon^{i}(1-\varepsilon)^{N-i} \leq \eta,
$$

where $d$ denotes the number of optimization variables in (2), then, if we set $k=\lfloor\alpha N \mid$, with probability no smaller than $1-\eta$, the solution $\left(A_{N, k}^{\star}, \boldsymbol{c}_{N, k}^{\star}\right)$ to the scenario optimization problem with constraint removal (2) satisfies $\mathcal{P}\{\delta \in \Delta$ : $\left.\boldsymbol{x}_{\delta}(t) \in \mathcal{E}_{\varepsilon}\left(A_{N, k}^{\star}, \boldsymbol{c}_{N, k}^{\star}\right)\right\} \geq 1-\varepsilon$.

Not surprisingly, feasibility of the scenario solution holds with a certain confidence $1-\eta$. This is because the scenario solution is a random quantity that depends on the extracted multi-sample $\delta^{(1)}, \delta^{(2)}, \ldots, \delta^{(N)}$. It may then happen that a bad multi-sample (e.g., all $\delta^{(i)}$ 's are equal) is extracted and the feasibility property does not hold. However, this event becomes more and more unlikely as $N$ increases and its probability $\eta$ can be set as small as $10^{-10}$ (i.e., zero in practice) without growing too much $N$. Indeed, when $\alpha=0$, the explicit bound $N \geq \frac{1}{\varepsilon}(d+1+\ln (1 / \eta)+\sqrt{2(d+1) \ln (1 / \eta)})$ in [32] shows that the dependence on $\eta$ is logarithmic.

Remark 1 (Choice of $\alpha$ ). Though the feasibility of the randomized solution is guaranteed for every $\alpha \in[0, \varepsilon)$, the closer $\alpha$ is to the desired violation probability $\varepsilon$, the better the randomized solution approximates the actual solution to the chance-constrained problem. At the same time, however, $N$ grows to infinity as $O\left(\frac{1}{\varepsilon-\alpha}\right)$ when $\alpha \rightarrow \varepsilon$, [29], so that one should choose $\alpha$ based on the available computational resources.

In order to extend the characterization of the debris dispersion to $4 \mathrm{D}$, we discretize the reference time horizon $\left[t_{i n}, t_{\text {out }}\right]$ and associate to each sampled time instant $t_{j}, j=1, \ldots, n_{s}$, an ellipsoidal set $\mathcal{E}_{\varepsilon}\left(A_{j}, \boldsymbol{c}_{j}\right), j=1, \ldots, n_{s}$. The sum of the volumes of all ellipses is then minimized subject to the constraint that a fraction of probability at least $1-\varepsilon$ of the debris trajectories belongs to the ellipsoidal sets:

$$
\min _{\left\{A_{j}, \boldsymbol{c}_{j}\right\}_{j=1}^{n_{s}}} \sum_{j=1}^{n_{s}} \log \operatorname{det} A_{j}^{-1}
$$

subject to:

$A_{j}=A_{j}^{T} \succ 0, j=1, \ldots, n_{s}$

$\mathcal{P}\left\{\delta \in \Delta: \boldsymbol{x}_{\delta}\left(t_{j}\right) \in \mathcal{E}_{\varepsilon}\left(A_{j}, \boldsymbol{c}_{j}\right), j=1, \ldots, n_{s}\right\} \geq 1-\varepsilon$

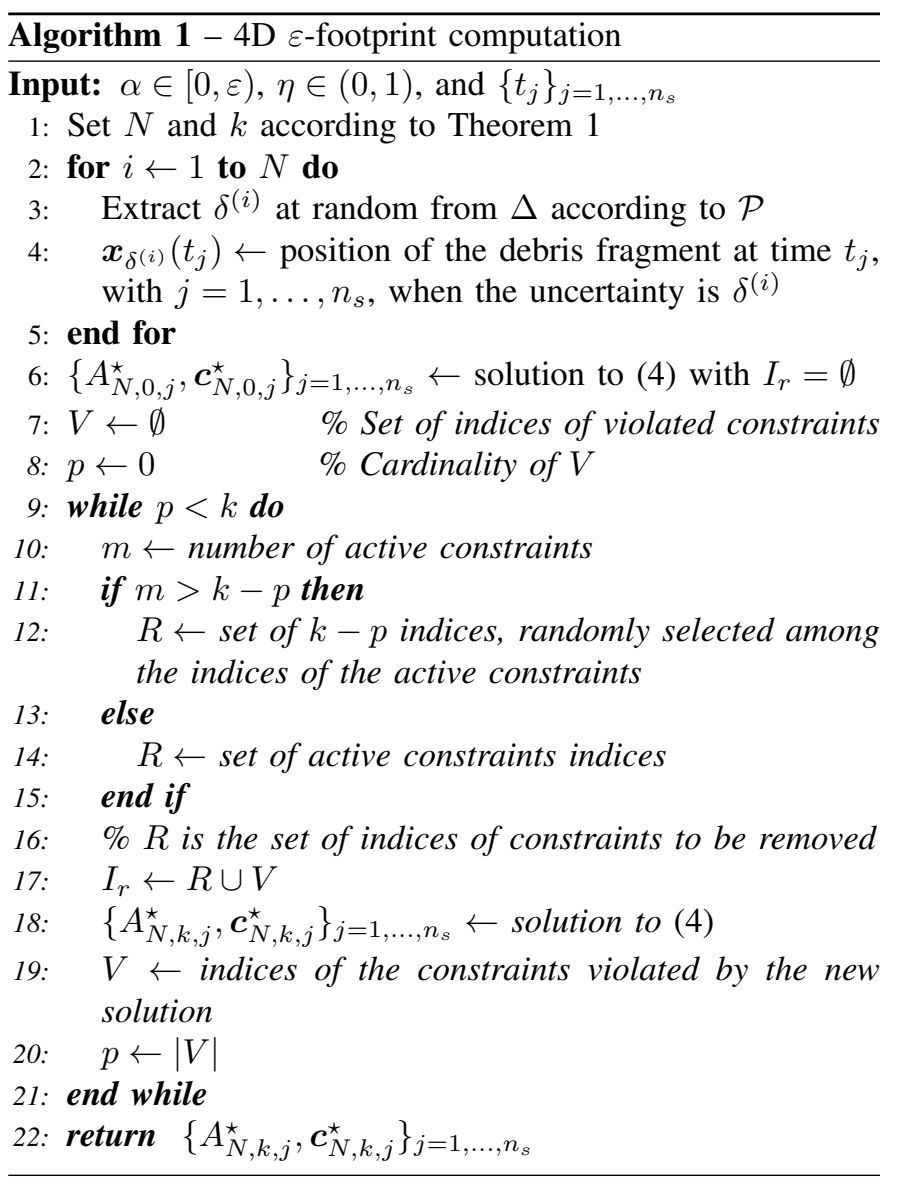

The scenario program with constraint removal

$\min _{\left\{A_{j}, \boldsymbol{c}_{j}\right\}_{j=1}^{n_{s}}} \sum_{j=1}^{n_{s}} \log \operatorname{det} A_{j}^{-1}$

subject to:

$A_{j}=A_{j}^{T} \succ 0, j=1, \ldots, n_{s}$

$\boldsymbol{x}_{\delta^{(i)}}\left(t_{j}\right) \in \mathcal{E}_{\varepsilon}\left(A_{j}, \boldsymbol{c}_{j}\right), j=1, \ldots, n_{s}, i \in\{1,2, \ldots, N\} \backslash I_{r}$,

can then be solved through Algorithm 1. Choosing the $k$ out of $N$ constraints to remove so as to obtain the best improvement in the cost is a combinatorial problem. However, since Theorem 1 holds irrespectively of the algorithm used to remove the constraints, one can opt for suboptimal approaches like removing one by one the constraint that leads to the largest improvement in the cost (greedy removal) or keeping removing the whole set of active constraints (block removal), i.e., those constraints such that $\left(\boldsymbol{x}_{\delta^{(i)}}(t)-\boldsymbol{c}\right)^{\top} A\left(\boldsymbol{x}_{\delta^{(i)}}(t)-\boldsymbol{c}\right)=1$. for the current $(A, c)$ solution. Algorithm 1 implements this latter removal procedure.

\section{Model of A ReEntering Debris Fragment}

To simulate the trajectory of a debris fragment after the breakup instant, we adopt the three-degrees of freedom model of a falling object over a rotating planet recently proposed in [15]. The reference coordinate system is named ENZ (EastNorth-Zenith) frame and is centered on the Earth surface at the (nominal) longitude $\vartheta_{0}$ and latitude $\varphi_{0}$ of the fragment at the breakup instant, with the $x_{3}$-axis directed towards the zenith 
and the $x_{1} x_{2}$ plane tangent to the Earth surface, the $x_{1}$-axis pointing eastward and the $x_{2}$-axis pointing northward. In this way, the initial position of the fragment at the breakup instant is $\boldsymbol{x}_{0}=\left[0,0, x_{3,0}\right]^{\top}$ with $x_{3,0}$ representing the nominal value for the altitude at which the breakup occurs. With reference to the ENZ frame, the equations of motion describing the debris trajectory are given by:

$$
\begin{aligned}
& \dot{\boldsymbol{x}}=\boldsymbol{v} \\
& \dot{\boldsymbol{v}}=-\boldsymbol{a}_{d}-g \boldsymbol{e}_{3}-2 \boldsymbol{\omega} \times \boldsymbol{v}-\boldsymbol{\omega} \times\left[\boldsymbol{\omega} \times\left(\boldsymbol{x}+R_{e} \boldsymbol{e}_{3}\right)\right]+\boldsymbol{\xi}
\end{aligned}
$$

where $\boldsymbol{x}=\left[x_{1}, x_{2}, x_{3}\right]^{\top}$ is the position, $\boldsymbol{v}=\left[v_{1}, v_{2}, v_{3}\right]^{\top}$ is the velocity, $\boldsymbol{a}_{d}$ is the atmospheric drag deceleration, $\boldsymbol{\omega}=$ $\left[0, \omega_{e} \cos \left(\varphi_{0}\right), \omega_{e} \sin \left(\varphi_{0}\right)\right]^{\top}$ is the angular velocity vector of the ENZ frame (Earth rotation rate: $\omega_{e}=7.2921 \times 10^{-5} \mathrm{rad} / \mathrm{s}$ ) and $e_{3}=[0,0,1]^{\top}$. Constant $R_{e}$ represents the Earth mean radius $\left(R_{e}=6.3728 \times 10^{6} \mathrm{~m}\right)$, whereas according to the inverse square gravity model, the gravitational acceleration is given by $g=g_{g}\left(R_{e} /\left(R_{e}+x_{3}\right)\right)^{2}$, where $g_{g}=9.81 \mathrm{~m} / \mathrm{s}^{2}$ is the gravitational acceleration on the ground $\left(x_{3}=0\right)$. The atmospheric drag deceleration $\boldsymbol{a}_{d}$ acting on the fragment can be expressed as $\boldsymbol{a}_{d}=\frac{1}{2} \frac{\rho\left(x_{3}\right)}{\beta} v_{r} \boldsymbol{v}_{r}$, where $\rho\left(x_{3}\right)$ is the atmosphere density as a function of the altitude, $\boldsymbol{v}_{r}=\boldsymbol{v}-\Lambda$ is the debris speed relative to the wind velocity $\Lambda$, and $v_{r}=\left\|\boldsymbol{v}_{r}\right\|$ is the magnitude of $\boldsymbol{v}_{r}$. Parameter $\beta$ represents the ballistic coefficient and is given by $\beta=m_{d} /\left(C_{d} \mathcal{A}\right)$, where $m_{d}$ is the mass of the debris, $\mathcal{A}$ its cross-sectional area, and $C_{d}$ the aerodynamics coefficient. Finally, $\boldsymbol{\xi}$ is an additive random acceleration vector that accounts for modeling errors and disturbances. The uncertainty elements can be collected in vector $\delta:=\left[\boldsymbol{x}_{0}^{\top} \boldsymbol{v}_{0}^{\top} \boldsymbol{\xi}^{\top} \Lambda^{\top} \beta\right]^{\top}$.

\section{COMPARATIVE AnAlysis}

The Covariance Propagation (CP) method proposed in [15] for determining the $4 \mathrm{D}$ footprint rests on the linearization of (5) around the nominal trajectory and on the description of the resulting perturbation as a Gauss-Markov process.

Set $\boldsymbol{s}=\left[\boldsymbol{x}^{\top}, \boldsymbol{v}^{\top}\right]^{\top}$. Then, (5) can be rewritten in the compact form

$$
\dot{s}=\boldsymbol{f}(\boldsymbol{s})+B \boldsymbol{\xi},
$$

where $B=\left[\begin{array}{ll}0_{3 \times 3} & I_{3 \times 3}\end{array}\right]^{\top}$. Now, define the perturbation vector $\boldsymbol{z}$ as $z=s-s_{n}$, where $s_{n}$ is the nominal trajectory obtained by neglecting $\boldsymbol{\xi}$ and the other sources of uncertainty affecting the system evolution. If we assume that the wind velocity vector $\Lambda$ entering (5) through the atmospheric drag deceleration $\boldsymbol{a}_{d}$ depends on the position only (i.e., $\Lambda=\Lambda(\boldsymbol{x})$ ), and set $A(t)=$ $\left.\frac{\partial \boldsymbol{f}}{\partial \boldsymbol{s}}\right|_{\boldsymbol{s}_{n}(t)}$, then, the linearised equations governing $\boldsymbol{z}$ are given by

$$
\dot{\boldsymbol{z}}=A(t) \boldsymbol{z}+B \boldsymbol{\xi} .
$$

Suppose that the disturbance vector $\boldsymbol{\xi}$ is a white Gaussian noise with mean and covariance given by $E[\boldsymbol{\xi}(t)]=$ $\overline{\boldsymbol{\xi}}(t)$ and $E\left[(\boldsymbol{\xi}(t)-\overline{\boldsymbol{\xi}}(t))(\boldsymbol{\xi}(t)-\overline{\boldsymbol{\xi}}(t))^{\top}\right]=\Xi(t)$, and that it is independent of the initial state at time $t_{i n}=0$. If $\boldsymbol{z}(0)$ is Gaussian $\boldsymbol{z}(0) \sim \mathcal{N}\left(\overline{\boldsymbol{z}}_{0}, Z_{0}\right)$, then, (7) describes a continuous time Gauss-Markov process with mean and covariance matrix $\overline{\boldsymbol{z}}(t)=E[\boldsymbol{z}(t)]$ and $Z(t)=$
$E\left[(\boldsymbol{z}(t)-\overline{\boldsymbol{z}}(t))(\boldsymbol{z}(t)-\overline{\boldsymbol{z}}(t))^{\top}\right]$, that satisfy $\dot{\overline{\boldsymbol{z}}}=A(t) \overline{\boldsymbol{z}}+$ $B \overline{\boldsymbol{\xi}}(t)$ and $\dot{Z}=A(t) Z+Z A(t)+B \Xi(t) B^{\top}$, initialized with $\bar{z}(0)=z_{0}$ and $Z(0)=Z_{0}$. The debris position at time $t$ can then be described as a Gaussian random variable with mean and covariance matrix $\overline{\boldsymbol{x}}(t)=C\left(\overline{\boldsymbol{z}}(t)+\boldsymbol{s}_{n}(t)\right)$ and $X(t)=C Z(t) C^{\top}$, where $C=\left[\begin{array}{ll}I_{3 \times 3} & 0_{3 \times 3}\end{array}\right]$.

As a consequence, the $3 \mathrm{D}$ ellipsoid containing a fraction $1-\varepsilon$ of the debris trajectories at time $t$ can be determined as an appropriate level set of the Gaussian distribution of $\overline{\boldsymbol{x}}(t)$, i.e., $\mathcal{E}_{\varepsilon}(t):=\left\{\boldsymbol{x} \in \mathbb{R}^{3}:[\boldsymbol{x}-\overline{\boldsymbol{x}}(t)]^{\top} X^{-1}(t)[\boldsymbol{x}-\overline{\boldsymbol{x}}(t)] \leq r_{\varepsilon}^{2}\right\}$, where $r_{\varepsilon}$ is the Mahalanobis distance between $\boldsymbol{x}$ and $\overline{\boldsymbol{x}}$ and can be computed as the $1-\varepsilon$ quantile of the $\chi^{2}$ distribution with 3 degrees of freedom: $\mathcal{P}\left(V \leq r_{\varepsilon}^{2}\right)=1-\varepsilon$ with $V \sim \chi^{2}(3)$.

The 4D footprint can then be obtained by varying $t$ within the reference time horizon $\left[t_{i n}, t_{\text {out }}\right]$ and considering the corresponding ellipsoidal set $\mathcal{E}_{\varepsilon}(t), t \in\left[t_{\text {in }}, t_{\text {out }}\right]$.

Remark 2 (approximation errors in the CP method). There are two sources of approximation in the evaluation of the $4 D$ footprint according to the outlined procedure: $i$ ) the footprint is constructed based on a linearized model of the system, and ii) the adopted level-set based procedure provides no guarantee that a fraction of probability $1-\varepsilon$ of the trajectories passes through all the ellipsoidal sets $\mathcal{E}_{\varepsilon}(t), t \in\left[t_{\text {in }}, t_{\text {out }}\right]$, e.g. the set of trajectories which pass through $\mathcal{E}_{\varepsilon}\left(t_{1}\right)$ may be different from that passing through $\mathcal{E}_{\varepsilon}\left(t_{2}\right), t_{\text {in }} \leq t_{1}<t_{2} \leq t_{\text {out }}$.

In order to compare the Simulation-Based (SB) method proposed in Section II with the CP method, we suppose that the only source of uncertainty is the initial state $s(0)$ at time $t_{i n}=0$ and consider the disturbance vector $\boldsymbol{\xi}(t)$ in (5) negligible, as in the simulation results presented in [15]. Differently from the simulation setting in [15], we use HWM93, [33], as nominal local wind model for both methods, and the U.S. Standard Atmosphere model [34] to obtain the atmospheric density in the range 0 to $120 \mathrm{~km}$. Given the geodetic coordinates (latitude, longitude and altitude) of a point in the Earth's atmosphere, HWM93 provides the mean value of wind speed along the eastward and northward directions. The wind speed component along the altitude direction is set equal to zero.

Results reported in this section refer to the case when the initial state $\boldsymbol{s}(0)$ is given by $\boldsymbol{s}(0)=\boldsymbol{s}_{n}(0)+$ $\boldsymbol{z}(0)$, where the nominal initial state is $\boldsymbol{s}_{n}(0)=$ $\left[\boldsymbol{x}_{n}(0)^{\top}, \boldsymbol{v}_{n}(0)^{\top}\right]^{\top}$, with $\boldsymbol{x}_{n}(0)=\left[0,0,7.8 \times 10^{4}\right]^{\top} \mathrm{m}$, $\boldsymbol{v}_{n}(0)=\left[7.0989 \times 10^{3}, 0,-123.9\right]^{\top} \mathrm{m} / \mathrm{s}$, and the perturbation to the nominal initial state $\boldsymbol{z}(0)$ is Gaussian with mean $\overline{\boldsymbol{z}}_{0}=0$ and covariance matrix $Z_{0}=\operatorname{blkdiag}\left(0_{3 \times 3}, V_{0}\right)$, where $V_{0}=\operatorname{diag}\left(\sigma_{v_{1}}^{2}, \sigma_{v_{2}}^{2}, \sigma_{v_{3}}^{2}\right)$, with $\sigma_{v_{1}}^{2}=\sigma_{v_{2}}^{2}=2500 \mathrm{~m}^{2} / \mathrm{s}^{2}$ and $\sigma_{v_{3}}^{2}=5300 \mathrm{~m}^{2} / \mathrm{s}^{2}$. All parameters are chosen to match the experiments reported in [15] for comparison purposes.

The time instants $\left\{t_{j}\right\}_{j=1, \ldots, n_{s}}$ for the 4D footprint calculation are determined by considering $n_{s}=10$ equally spaced samples of the nominal trajectory $\boldsymbol{s}_{n}(t), t \in\left[t_{\text {in }}, t_{\text {out }}\right]$, along the $x_{3}$-axis (i.e. the altitude in the ENZ reference frame). Different values for the violation parameter $\varepsilon$ and for the empirical violation $\alpha$ are considered, whereas the confidence parameter $\eta$ is set equal to $\eta=10^{-5}$ in all simulations. The value for $N$ satisfying the bound in Theorem 1 depend on the 
considered $(\varepsilon, \alpha, \eta)$, the maximal $N$ being $N=10780$.

Table I summarises the results obtained with the SB method and the $\mathrm{CP}$ method for a set of values of $\varepsilon$. The value for $\alpha$ used in the SB method is specified in the second column. In all simulations we maintained fixed $N=10780$ and removed $k=\lfloor\alpha N\rfloor$ constraints.

The comparison between the SB method and CP method is in terms of i) volume $\mathcal{V}$ of the corresponding $4 \mathrm{D}$ footprints (reported in $\mathrm{km}^{3}$ in the last two columns of Table I) and ii) actual violation $\hat{\varepsilon}$ computed via Monte Carlo simulation by generating a further set of $N$ simulated trajectories and evaluating the fraction of them that exits the 4D footprint.

TABLE I

COMPARISON BETWEEN SB AND CP METHODS IN TERMS OF ACTUAL VIOLATION $\hat{\varepsilon}$ AND 4D FOOTPRINT VOLUME $\mathcal{V}$.

\begin{tabular}{c|c||c|c|c|c}
\hline$\varepsilon$ & $\alpha$ & $\hat{\varepsilon}_{N}^{S B}$ & $\hat{\varepsilon}_{N}^{C P}$ & $\mathcal{V}^{S B}$ & $\mathcal{V}^{C P}$ \\
\hline 0.500 & 0.350 & 0.3505 & 0.5933 & 452.47 & 235.10 \\
0.400 & 0.260 & 0.2602 & 0.5083 & 625.91 & 326.69 \\
0.300 & 0.170 & 0.1705 & 0.4261 & 907.73 & 453.24 \\
0.200 & 0.100 & 0.1003 & 0.3366 & 1304.18 & 646.03 \\
0.100 & 0.035 & 0.0353 & 0.2370 & 2208.56 & 1009.74 \\
0.050 & 0.010 & 0.0103 & 0.1724 & 3552.52 & 1411.29 \\
0.025 & 0.002 & 0.0020 & 0.1328 & 5494.42 & 1846.50 \\
0.020 & 0.001 & 0.0012 & 0.1224 & 6845.50 & 1993.27 \\
0.015 & 0 & $<5 \cdot 10^{-5}$ & 0.1109 & 9182.69 & 2187.03 \\
\hline
\end{tabular}

Note that if we look at each single row of Table I, the 4D footprint volume $\mathcal{V}^{S B}$ obtained with the SB method is larger than volume $\mathcal{V}^{C P}$ obtained with the CP method. However, the violation $\hat{\varepsilon}^{C P}$ of the $\mathrm{CP}$ method always exceeds the desired $\varepsilon$ value (possibly due to the approximations errors involved in the method, see Remark 2), whereas the violation $\hat{\varepsilon}^{S B}$ of the SB method is always smaller. If we make a comparison between the volume of the 4D footprints corresponding to the same actual violation (i.e., $\hat{\varepsilon}^{C P} \simeq \hat{\varepsilon}^{S B}$ ), the SB method outperforms the $\mathrm{CP}$ one. For instance, considering the third and sixth rows, $\hat{\varepsilon}^{S B}=0.1705$ and $\mathcal{V}^{S B}=907.73$ (third row) and $\hat{\varepsilon}^{C P}=0.1724 \simeq \hat{\varepsilon}^{S B}$ and $\mathcal{V}^{C P}=1411.29 \gg \mathcal{V}^{S B}$ (sixth row). As for the SB method, it is worth noticing that actual violation $\hat{\varepsilon}_{N}^{S B}$ is very close to the chosen empirical violation $\alpha$, which affects the size of the $4 \mathrm{D}$ footprint.

As a final remark, the SB method has the additional advantage with respect to the $\mathrm{CP}$ method of being applicable to a more general setting, where further sources of uncertainties (like that on the ballistic coefficient or the local wind) are present besides that on the initial velocity. As a matter of fact, wind can play a main role and cause a significant increase of the footprint volume. The numerical results in Section VI show that by exploiting radar measurements of the aircraft position, uncertainty on the wind can be reduced and the footprint volume significantly decreased.

\section{Footprint Refinement Via Nonlinear FiLtering}

In this section, we address the problem of using available measurements of the debris fragment and aircraft positions so as to improve the estimate of the debris fragment probabilistic footprint. The proposed approach involves the adoption of nonlinear filtering techniques and their integration in the footprint calculation. The idea is to use the Unscented Kalman Filter (UKF) so as to determine the a-posteriori probability of the debris state given the measurements of its position, and to run simultaneously a sequential version of the particle filter so as to gather information from the aircraft radar measurements on the actual wind field and improve the wind forecast.

\section{A. Debris State Estimation}

The Extended Kalman Filter (EKF) addresses state estimation for nonlinear systems by propagating the mean and covariance of the state estimate through the linearized system dynamics. As a consequence, its performance typically turns out to be unsatisfactory in presence of strong nonlinearities. In the UKF in [11], the so-called Unscented Transformation (UT) is introduced to overcome this limitation. Interestingly, in [11], the UKF is shown to better perform than the EKF in a numerical example similar to our debris state estimation problem. The basic idea of the UT is to approximate the probability distribution of the state by a set of weighted sigma points matching its mean and covariance, and then propagate these points through the nonlinear dynamics. More precisely, let $\mu_{t}$ and $\Sigma_{t}$ be the mean and covariance of the debris state $\boldsymbol{s}=\left[\boldsymbol{x}^{\top}, \boldsymbol{v}^{\top}\right]^{\top}$ at time $t$, and suppose we are using $p+1$ sigma points $\boldsymbol{s}_{t}^{(i)}, i=0,1, \ldots, p$. If we denote by $W_{\mu}^{(i)}$ and $W_{\Sigma}^{(i)}, i=0,1,2, \ldots, p$, their weights, then, it holds that $\mu_{t}=$ $\sum_{i=0}^{p} W_{\mu}^{(i)} \boldsymbol{s}_{t}^{(i)}$ and $\Sigma_{t}=\sum_{i=0}^{p} W_{\Sigma}^{(i)}\left[\boldsymbol{s}_{t}^{(i)}-\mu_{t}\right]\left[\boldsymbol{s}_{t}^{(i)}-\mu_{t}\right]^{\top}$.

Among the possible choices for the set of sigma points and their weights, we adopt the one in [35] given by

$$
\boldsymbol{s}_{t}^{(0)}=\mu_{t}, \boldsymbol{s}_{t}^{(i)}=\mu_{t}+a \sqrt{n}\left(\Gamma_{t}\right)_{i}, \boldsymbol{s}_{t}^{(i+n)}=\mu_{t}-a \sqrt{n}\left(\Gamma_{t}\right)_{i},
$$

with $i=1, \ldots, n$, where $n=6$ is the dimension of the debris state vector, and $\left(\Gamma_{t}\right)_{i}$ is the $i^{t h}$-row of the matrix $\Gamma_{t}$ obtained by performing a Cholesky decomposition of $\Sigma_{t}$, i.e., $\Sigma_{t}=$ $\Gamma_{t}^{\top} \Gamma_{t}$. In this way, $\boldsymbol{s}_{t}^{(i)}, i=1, \ldots, 2 n$, are positioned on some ellipsoidal set with shape matrix $\Sigma_{t}$ and center $\mu_{t}$, at a distance from the center that can be tuned via $a \in(0,1]$. As for the weights, we set

$$
\begin{aligned}
& W_{\mu}^{(0)}=1-\frac{1}{a^{2}}, \quad W_{\Sigma}^{(0)}=1-\frac{1}{a^{2}}+\left(1-a^{2}+b\right) \\
& W_{\mu}^{(i)}=W_{\Sigma}^{(i)}=\frac{1}{2 a^{2}},
\end{aligned}
$$

where $a \in(0 ; 1]$ and $b \geq 0$ are scaling parameters and the resulting UT is named Scaled UT (SUT). Parameter $a$ controls the distance of the sigma points from their mean, and both $a$ and $b$ can be tuned to exploit possibly available information on higher order moments. Precisely, $a$ can be set small enough so as to reduce the approximation errors of the mean and the covariance matrix. As for $b, b=2$ is the optimal value for minimizing the approximation error of the covariance matrix when the probability distribution is Gaussian, [35].

In the UKF, the debris dynamics is propagated for $d t$ time instants starting from each sigma point $\boldsymbol{s}_{t}^{(i)}$. The resulting $\hat{\boldsymbol{s}}_{t+d t}^{(i)}$ is named transformed sigma point. Updated estimates $\tilde{\mu}_{s}$ and $\tilde{\Sigma}_{s}$ of mean and covariance matrix of the a-priori 


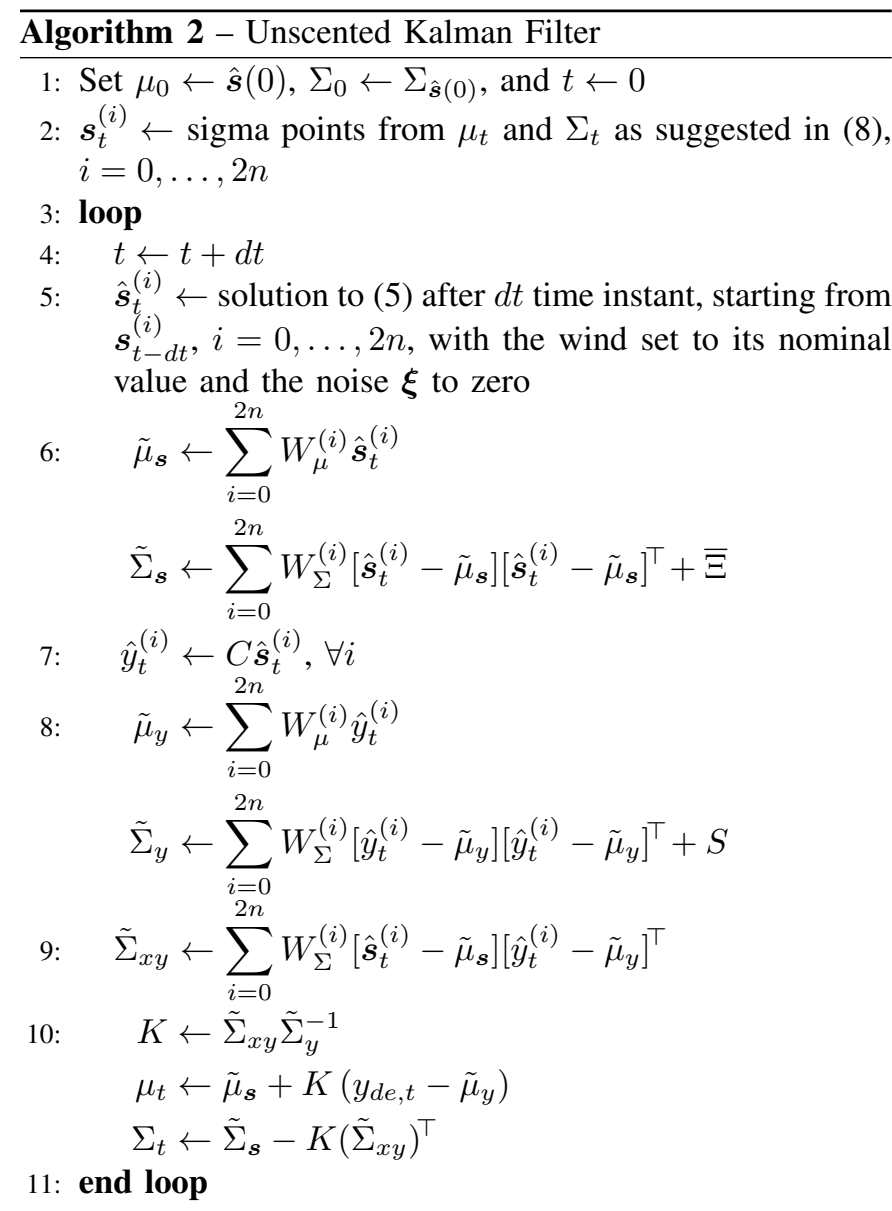

distribution of the debris state vector at time $t+d t$ can be derived from the transformed sigma points set according to $\tilde{\mu}_{\boldsymbol{s}}=\sum_{i=0}^{p} W_{\mu}^{(i)} \hat{\boldsymbol{s}}_{t+d t}^{(i)}$ and $\tilde{\Sigma}_{\boldsymbol{s}}=\sum_{i=0}^{p} W_{\Sigma}^{(i)}\left[\hat{\boldsymbol{s}}_{t+d t}^{(i)}-\right.$ $\left.\tilde{\mu}_{\boldsymbol{s}}\right]\left[\hat{\boldsymbol{s}}_{t+d t}^{(i)}-\tilde{\mu}_{\boldsymbol{s}}\right]^{\top}+\bar{\Xi}$, where $\bar{\Xi}$ is the covariance matrix of the discretized noise in (6).

Let $y_{d e, t+d t}=C \boldsymbol{s}_{t+d t}+n_{t+d t}$ be the measurement of the debris position at time $t+d t$, where $C=\left[\begin{array}{ll}I_{3 \times 3} & 0_{3 \times 3}\end{array}\right]$ and $n_{t}$ is a zero mean Gaussian noise with covariance matrix $S$. Then, mean and covariance matrix of the a-posteriori distribution of the debris state vector at time $t+d t$ can be computed by means of the standard KF equations.

The overall procedure described above is reported in Algorithm 2, which is a slightly simplified version of the original UKF presented in [11]. Algorithm 2 uses the SUT in place of the UT, and exploits the fact that the nonlinear debris dynamics in Section III has additive Gaussian process noise with zero mean and covariance matrix $\Xi$. The algorithm takes as inputs some a-priori estimate $\hat{\boldsymbol{s}}(0)$ and covariance $\Sigma_{\hat{\boldsymbol{s}}(0)}$, and then applies the UKF online each time a new measurement $y_{d e}$ of the debris position is available. Note that the UKF can be used to estimate uncertain parameters. Indeed, in Section VI, we use the UKF to estimate the ballistic coefficient $\beta$. This involves extending the debris state vector to include $\beta$ and attributing to $\beta$ the trivial dynamics $\dot{\beta}=0$.

\section{B. Wind Estimation}

The wind velocity $\Lambda$ affects the debris motion (5) through the atmospheric drag deceleration $\boldsymbol{a}_{d}$. Wind velocity is uncertain and this causes a dispersion of the debris trajectories and an enlarged footprint. The idea developed here is to improve the footprint estimate obtained via Algorithm 1 by using aircraft as sensors to reduce the uncertainty on $\Lambda$. More specifically, we follow the approach in [12] where a Sequential Conditional Particle Filter (SCPF) is introduced to estimate the wind field in a certain region of the airspace based on radar measurements of the aircraft position. We next briefly describe the contributions in [12] that are relevant to our context.

1) Aircraft Model: The aircraft model developed in [12], [36], [37] is a stochastic hybrid system [38], which comprises a continuous dynamics describing the physical motion of the aircraft, a discrete dynamics representing the flight management system (FMS), and a stochastic component given by the wind affecting the aircraft motion. The following point mass model is adopted for the aircraft motion

$$
\left[\begin{array}{c}
\dot{X}_{1} \\
\dot{X}_{2} \\
\dot{X}_{3} \\
\dot{V} \\
\dot{\psi} \\
\dot{m}
\end{array}\right]=\left[\begin{array}{c}
V \cos (\psi) \cos (\gamma)+\lambda_{1} \\
V \sin (\psi) \cos (\gamma)+\lambda_{2} \\
V \sin (\gamma)+\lambda_{3} \\
\frac{T-D}{m}-g \sin (\gamma)-\Lambda_{\text {agf }} \cos (\gamma) V \sin (\gamma) \\
\frac{1}{m} \frac{L \sin (\varphi)}{V \cos (\gamma)}-\Lambda_{\mathrm{cgf}} \tan (\gamma) \\
-\eta_{c} T
\end{array}\right]
$$

where $X_{1}, X_{2}$ and $X_{3}$ are the coordinates of the aircraft position with respect to an inertial reference $X_{1, r}, X_{2, r}$, $X_{3, r}$ with origin fixed at some point on Earth's surface (i.e. the radar position), $V$ is the true air speed, $\psi$ is the heading angle, and $m$ is the mass. Wind acts as a disturbance and enters the aircraft dynamics additively through its speed $\Lambda=\left(\lambda_{1}, \lambda_{2}, \lambda_{3}\right) \in \mathbb{R}^{3}$. The bank angle $\varphi$, the flight path angle $\gamma$, and the engine thrust $T$ are treated as inputs (i.e. commands from pilot for directing the aircraft). The lift and drag forces $L$ and $D$ can be expressed as canonic-form aerodynamics forces as follows: $L=\frac{1}{2} C_{L} S_{w} \rho\left(X_{3}\right) V^{2}$ and $D=\frac{1}{2} C_{D} S_{w} \rho\left(X_{3}\right) V^{2}$, where $S_{w}$ is the total wings surface area, $\rho\left(X_{3}\right)$ the air density at altitude $X_{3}$, and $C_{D}$ and $C_{L}$ are the drag and lift coefficients, which depend on the angle of attack AOA and the side slip angle AOS. The engine thrust $T$ affects mass variation through $\eta_{c}$ that represents the fuel consumption rate. As for $\Lambda_{\text {agf }}$ and $\Lambda_{\text {cgf }}$ appearing in (10), they represent the along-track and cross-track wind gradient factors and are given by $\Lambda_{\text {agf }}=\frac{\partial \lambda_{1}}{\partial X_{3}} \cos (\psi)+\frac{\partial \lambda_{2}}{\partial X_{3}} \sin (\psi)$ and $\Lambda_{\mathrm{cgf}}=\frac{\partial \lambda_{1}}{\partial X_{3}} \sin (\psi)+\frac{\partial \lambda_{2}}{\partial X_{3}} \cos (\psi)$. The parameters entering the dynamics depend on the type of aircraft and can be retrieved from the Base of Aircraft Data (BADA) documents, [39]. If the aircraft is flying at constant altitude and with constant speed (i.e. trimmed flight), then, $X_{3}$ and $V$ are held constant by setting $\gamma$ equal to 0 and the trust $T$ equal to $D$, respectively. We can hence drop the third and fourth equations in (10) and set $\mathrm{AOA}=\mathrm{AOS}=0,[12]$. This entails that equations (10) 
simplify to

$$
\left[\begin{array}{c}
\dot{X}_{1} \\
\dot{X}_{2} \\
\dot{\psi} \\
\dot{m}
\end{array}\right]=\left[\begin{array}{c}
V \cos (\psi)+\lambda_{1} \\
V \sin (\psi)+\lambda_{2} \\
\frac{1}{2 m} \rho\left(X_{3}\right) C_{L} S_{w} V \sin (\varphi) \\
-\frac{1}{2} \eta_{c} C_{D} S_{w} \rho\left(X_{3}\right) V^{2}
\end{array}\right] .
$$

The only input to set is then $\varphi$, which is set by the FMS so as to track the reference path by compensating cross-track deviations.

Aircraft positions are obtained from radar measurements. Radar noise is supposed to affect only the $X_{1}$ and $X_{2}$ measurements, and the altitude at which the aircraft is flying is supposed to be constant and known. The accuracy of the radar decreases as an aircraft moves away from its location. One can however use a sufficiently large value for the standard deviation $\sigma_{r}$ of the radar error measurements and keep it constant.

2) Wind Modeling: The wind velocity entering equations (10) is modeled as a random field composed by two contributions: the weather forecast (the nominal part) and the forecast error (the stochastic part).

The stochastic wind field component is supposed to satisfy the following conditions:

- the vertical component of the wind is zero;

- the horizontal components $\lambda_{1}(t, p)$ and $\lambda_{2}(t, p)$ in the $X_{1}$ (eastward) and $X_{2}$ (northward) directions associated with time $t \in \mathbb{R}$ and position $p \in \mathbb{R}^{3}$ are independent Gaussian random variables with zero mean;

- the wind field is isotropic (invariant under rotations) in the horizontal plane;

- the wind variance depends only on the altitude.

Given these assumptions, we can characterize the stochastic wind field through the correlation function

$$
\begin{aligned}
& r\left(t, p, t^{\prime}, p^{\prime}\right)=E\left[\lambda_{1}(t, p) \lambda_{1}\left(t^{\prime}, p^{\prime}\right)\right]=E\left[\lambda_{2}(t, p) \lambda_{2}\left(t^{\prime}, p^{\prime}\right)\right] \\
& =\sigma\left(X_{3}\right) \sigma\left(X_{3}^{\prime}\right) r_{t}(\tau) r_{12}\left(d_{12}\right) r_{3}\left(\left|\varrho\left(X_{3}\right)-\varrho\left(X_{3}^{\prime}\right)\right|\right)
\end{aligned}
$$

where $t, t^{\prime} \in \mathbb{R}, p=\left(X_{1}, X_{2}, X_{3}\right), p^{\prime}=\left(X_{1}^{\prime}, X_{2}^{\prime}, X_{3}^{\prime}\right) \in \mathbb{R}^{3}$, $\tau=\left|t-t^{\prime}\right|, d_{12}=\left\|\left[\left(X_{1}-X_{1}^{\prime}\right)^{\top} \quad\left(X_{2}-X_{2}^{\prime}\right)^{\top}\right]^{\top}\right\|, \varrho\left(X_{3}\right)$ being the atmospheric pressure and $r(t, p, t, p)=\sigma\left(X_{3}\right)^{2}$ the variance of the wind at altitude $X_{3}$. Quantities $\sigma\left(X_{3}\right), r_{t}$, $r_{12}$ and $r_{3}$ are defined in [12], so as to match the empirical data in [40]. Wind correlation decays exponentially with the distance in the horizontal plane, with the pressure difference in the vertical direction, and with time separation. In particular, the wind field is characterized by a strong time correlation, a significant but smaller horizontal correlation, and a very weak altitude correlation. In a 30 minutes look-ahead time horizon, the following expression holds for the time correlation $r_{t}$ : $r_{t}\left(\left|t-t^{\prime}\right|\right) \approx e^{-\left|t-t^{\prime}\right| / G_{t}}$, where $G_{t}$ is an appropriate constant, see [12].

In the SCPF, we need to generate wind realizations consistent with the above described structure. To this purpose, the airspace region of interest is discretized into $N_{1}, N_{2}$, and $N_{3}$ bins along the three orthogonal axes with bin size $d_{1}, d_{2}$ and $d_{3}$, respectively, while the look-ahead time horizon is split into $N_{t}$ time-steps of size $d t$. At each time step $t=k d t$, with $k \in\left\{0 \ldots N_{t}\right\}$, we can then define the two vectors $\Lambda_{1}(k)$ and $\Lambda_{2}(k)$ containing the horizontal components $\lambda_{1}(t, p)$ and $\lambda_{2}(t, p)$ sampled on the spatial grid. Due to the isotropy assumption, $\Lambda_{1}$ and $\Lambda_{2}$ have the same covariance matrix $R$, which can be derived based on the correlation function (12). Finally, wind realizations can be generated according to the following linear equations:

$$
\left\{\begin{array}{l}
\Lambda_{1}(k+1)=a \Lambda_{1}(k)+Q \zeta_{1}(k+1) \\
\Lambda_{2}(k+1)=a \Lambda_{2}(k)+Q \zeta_{2}(k+1)
\end{array}\right.
$$

initialized with $\Lambda_{1}(0)=\hat{Q} \zeta_{1}(0)$ and $\Lambda_{2}(0)=\hat{Q} \zeta_{2}(0)$, where $\zeta_{1}(k), \zeta_{2}(k)$ are standard independent Gaussian random variables, and matrices $\hat{Q}$ and $Q$ are derived by using the Cholesky decomposition of $R$ and satisfy $\hat{Q} \hat{Q}^{\top}=R$ and $Q Q^{\top}=\left(1-a^{2}\right) R$ with $a=e^{-d t / G_{t}}$. These choices are such that the covariance matrices of $\Lambda_{1}$ and $\Lambda_{2}$ in (13) are constant and equal to $R$ (see the appendix of [12] for a proof). The wind at some point that does not belong to the grid is computed by linear interpolation of $\Lambda_{1}$ and $\Lambda_{2}$.

3) Sequential Conditional Particle Filter: The SCPF introduced in [12] is adopted here to derive the conditional probability distribution of the wind vectors $\Lambda_{1}$ and $\Lambda_{2}$ in some region of interest, given the radar measurements of the positions of the aircraft flying in that region. The idea is that aircraft can be used as flying sensors to obtain an indirect local measure of the wind, which can then be extended to all the grid points thanks to the spatial correlation structure of the wind field. Note that the wind evolution is governed by linear equations fed by a Gaussian noise (see (13)) so that if measurements of $\Lambda_{1}$ and $\Lambda_{2}$ were available, then, standard Kalman filtering could be applied to compute mean and covariance matrix of their Gaussian conditional distributions. In the SCPF, such measurements are reconstructed from the measurements of the aircraft positions by implementing a PF, which estimates the conditional distribution of the aircraft state vectors via a set of particles. These particles contain also realizations of $\Lambda_{1}$ and $\Lambda_{2}$. In the filtering step, particles are resampled based on their likelihood as derived from the radar measurements. The resampled wind realizations are then used as local measurements of $\Lambda_{1}$ and $\Lambda_{2}$ to update mean and covariance matrix of the wind Gaussian conditional distributions associated to each particle, via a Kalman filter. Due to the fact that, conditioned to the wind field, the aircraft dynamics are decoupled, the filtering step can be performed one aircraft at a time, hence the term "sequential".

\section{Improved Footprint Estimate and No-Fly Zones}

The UKF for the debris state estimation in Section V-A and the SCPF for the wind field estimation in Section V-B can be jointly exploited to improve the debris footprint. More precisely, air traffic controllers can run the SCPF continuously so as to have a constantly updated estimate of the wind field over their sector. When a debris reentry occurs and a measurement of the debris position becomes available, the UKF can be activated to process a certain (limited) number of debris measurements before generating the footprint estimate 
via the simulation-based Algorithm 1 in Section II. The algorithm uses the last a-posteriori mean and covariance matrix of the debris state obtained via the UKF and the a-posteriori mean and covariance matrix of the wind field obtained via the SCPF to generate realizations of the debris trajectories. The dispersion of the trajectories is thus reduced and the debris footprint enhanced. By intersecting the footprint with planes at different flight levels, air traffic controllers can obtain nofly zones forbidden to air traffic in certain time frames and appropriately re-route aircraft so as to ensure their safety. Note that the larger a no-fly zone is, the more difficult it is to find a feasible solution to the problem of re-routing the aircraft. $\varepsilon$ can then play the role of a tuning parameter so as to compromise between the admissible risk level and the size of the no-fly zone: 0 risk but a large no-fly zone if $\varepsilon=0$, and maximum risk but a zero volume no-fly zone if $\varepsilon=1$.

\section{A Numerical ExAmple}

We next apply the proposed method for debris footprint estimation to a reference scenario and demonstrate its effectiveness. Aircraft and wind parameters are set as in [12].

We consider 6 aircraft flying at constant altitude in a region of the airspace of $600 \mathrm{~km}$ by $600 \mathrm{~km}$ in the $X_{1} X_{2}$ plane and the 9-12 km range along $X_{3}$. The flight plan of each aircraft is composed of a starting waypoint and a target waypoint. The aircraft state vectors are initialized with the corresponding starting waypoints, a nominal speed of $214 \mathrm{~m} / \mathrm{s}$, an initial mass of 46 tonnes, and a heading angle that points towards the corresponding target waypoint. All aircraft are assumed to be Boeing 737-700, and their parameters are taken from BADA, [39]. Figure 1 represents the aircraft by either a dot or a diamond, and flight plans as straight lines. Aircraft are divided in two sets: the first set contains 3 aircraft flying from the south-west corner of the airspace region to the north-east corner, while the other 3 aircraft in the second set fly from the north-west to the south-east corner. Nominal trajectories cross at the center of the considered airspace region, but aircraft reaching the central point at the same time are actually flying at different altitudes. More specifically, the aircraft marked with a dot in Figure 1 fly at altitude $10.5 \mathrm{~km}$ and the other ones at altitude $11.5 \mathrm{~km}$.

To simulate the wind field, we consider a grid whose blocks have dimensions $d_{1}=60 \mathrm{~km}, d_{2}=60 \mathrm{~km}$, and $d_{3}=1 \mathrm{~km}$ along the $X_{1}, X_{2}$, and $X_{3}$ directions respectively. In each point of the grid, the $X_{1}$ and $X_{2}$ components of the wind velocity is the sum of two contributions: a forecast value obtained from the HWM93 model, [33], plus a stochastic component that is generated according to equations (13) presented in Section V-B2 at altitudes between 9 and $12 \mathrm{~km}$. Each aircraft state vector then evolves according to equations (11) (see Section V-B1), where the air density is given by the U.S. Standard Atmosphere, [34], and the bank angle $\varphi$ is set by a nonlinear feedback controller that reduces the cross-track deviation with respect to the flight plan, [37]. We will refer to these trajectories as the "true" ones. The aircraft radar positions are obtained by sampling these trajectories every $d t=30$ seconds and adding a zero mean Gaussian noise with variance $\sigma_{r}^{2}=6400 \mathrm{~m}^{2}$ to each sample.

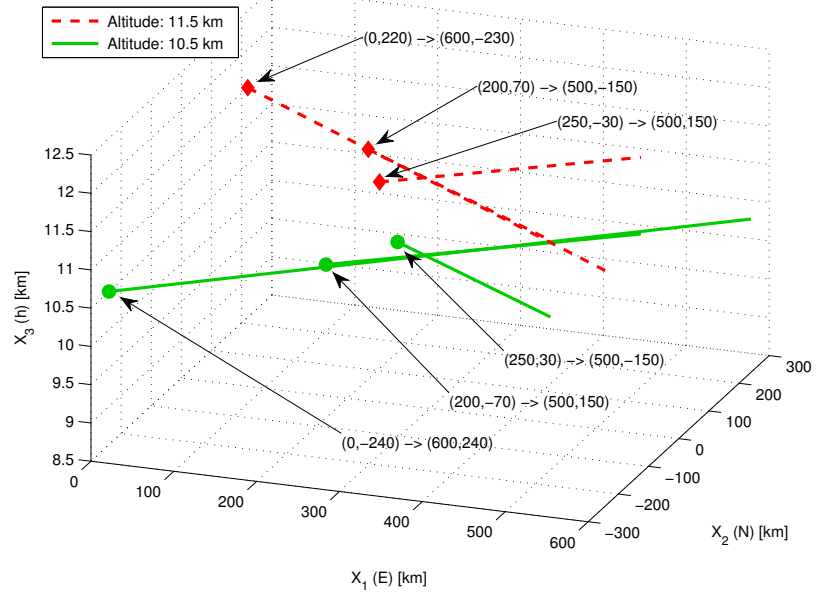

Fig. 1. Air traffic configuration: aircraft flying at the same altitude are marked with the same symbol (diamond or dot) and the same line for their nominal path (dotted or solid line).

After 15 minutes, a debris object enters the Earth's atmosphere. Its initial (extended) state is extracted at random from a Gaussian distribution with mean $\overline{\boldsymbol{s}}(0)=\left[\overline{\boldsymbol{x}}(0)^{\top}, \overline{\boldsymbol{v}}(0)^{\top}, \bar{\beta}\right]^{\top}$ and covariance matrix $\Sigma_{0}=\operatorname{diag}\left(\sigma_{d}^{2}, \sigma_{d}^{2}, \sigma_{d}^{2}, \sigma_{v_{1}}^{2}, \sigma_{v_{2}}^{2}, \sigma_{v_{3}}^{2}, \sigma_{\beta}^{2}\right)$, where $\overline{\boldsymbol{x}}(0)=$ $\left[0,0,7.8 \times 10^{4}\right]^{\top} \mathrm{m}, \overline{\boldsymbol{v}}(0)=\left[7.0989 \times 10^{3}, 0,-123.9\right]^{\top} \mathrm{m} / \mathrm{s}$, $\beta=5 \mathrm{~kg} / \mathrm{m}^{2}, \sigma_{d}^{2}=25600 \mathrm{~m}^{2}, \sigma_{v_{1}}^{2}=\sigma_{v_{2}}^{2}=2500 \mathrm{~m}^{2} / \mathrm{s}^{2}$, $\sigma_{v_{3}}^{2}=5300 \mathrm{~m}^{2} / \mathrm{s}^{2}$, and $\sigma_{\beta}^{2}=0.01 \mathrm{~kg}^{2} / \mathrm{m}^{4}$. The debris state vector evolves according to equations (5) subject to the wind field and to a random accelerations process $\boldsymbol{\xi}(t)$, which is a Gaussian white noise with zero mean and covariance matrix $\Xi=2.4064 \cdot 10^{-5} \cdot I_{3 \times 3}$, as suggested in [11]. We will refer to this trajectory as the "true" debris trajectory. The debris radar measurements are obtained by sampling the true positions every $d t=30$ seconds and adding a zero mean Gaussian noise with variance $\sigma_{d}^{2}=25600 \mathrm{~m}^{2}$.

We first apply the SCPF for wind estimation based on the radar measurements of the aircraft position. We used 500 particles in the SCPF implementation. The state vector of the $i$-th aircraft in all the particles is initialized with the first radar measurement of the corresponding aircraft, assuming the heading angle $\psi$ and the mass $m$ known. The wind field initial distribution is assumed to have a zero mean (in both the $X_{1}$ and the $X_{2}$ direction) and a covariance matrix equal to $R$, as described in Section V-B2. The SCPF is run for 15 minutes, after which the first measurement of the debris position becomes available. When this happens, we initialize the UKF as follows: the measured position is used to initialize the first three components of the state vector, the rest of the initial state vector and the initial covariance matrix are set to $\overline{\boldsymbol{v}}(0), \bar{\beta}$ and $\Sigma_{0}$, according to the reference scenario. As for the measurement noise covariance matrix, we set $S=\sigma_{d}^{2} \cdot I_{3 \times 3}$. When after 30 seconds another measurement of the debris position is available, a first iteration of the UKF is run with the parameters $a$ and $b$ in (9) set equal to $a=0.001$ and $b=2$. Both SCPF and UKF filters are then stopped. The UKF returns the a-posteriori mean and covariance matrix of the debris state vector, while the SCPF returns a set of particles 

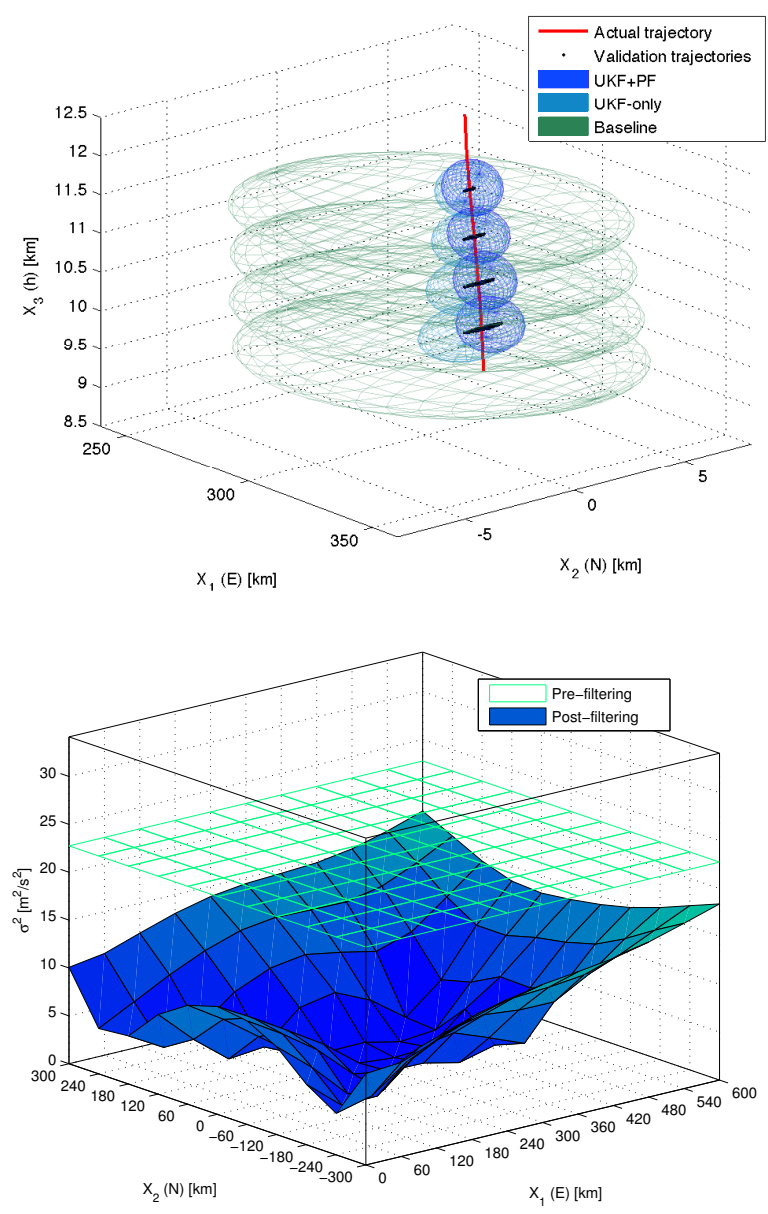

Fig. 2. Top: footprint comparison. Bottom: wind field covariance matrix before and after filtering.

which include a-posteriori mean and covariance matrix of the stochastic wind field among their components. Algorithm 1 is finally run for the footprint computation. We set $\varepsilon=0.1$, $\alpha=0.035, \eta=10^{-5}$ and $N$ according to Theorem 1 . The initial states of the $N$ trajectories are extracted according to the a-posteriori mean and covariance matrix of the debris state vector returned by the UKF. The particles returned by the SCPF are instead used to generate $N$ realizations of the stochastic component of wind field that will affects the $N$ extracted debris trajectories.

For comparison purposes, we also report the results obtained from Algorithm 1 in case no information about the wind field is available (i.e., the SCPF is not implemented) and in case no filtering procedure is applied (i.e., both SCPF and UKF are not implemented) as a baseline. The resulting footprints are shown in Figure 2 (top plot). The corresponding volumes are $2613.22 \mathrm{~km}^{3}$ (Baseline), $111.67 \mathrm{~km}^{3}$ (UKF-only) and 80.13 $\mathrm{km}^{3}$ (UKF+PF). Ellipsoids of the same color represent 3D hazardous region in four different time instants. Not surprisingly, the largest footprint is the one obtained without any filtering procedure, the intermediate one is that computed using only the UKF filtering procedure, and the smallest one is obtained using both UKF and SCPF. As can be seen, the volume of the footprint is progressively reduced as more information are incorporated via the two filtering procedures. In particular, the UKF filtering reduces the footprint volume by $95.72 \%$ with respect to the baseline, and if the SCPF is introduced as well, then the footprint volume is further reduced by an additional $28.25 \%$. This volume reduction is easily justified if one considers that the UKF is reducing the uncertainty on the debris position, and the SCPF is reducing the uncertainty on the wind forecast errors. Figure 2 (bottom plot) represents the variance of the wind speed vector associated with the grid points $\left(X_{1}, X_{2}\right)$ at the altitude of $11 \mathrm{~km}$ before and after applying the SCPF. As can be seen, after 15 minutes only, wind variances have been successfully lowered by almost a factor of two in those region of the airspace where radar measurements of the aircraft position are available. Finally, it is worth noticing that, as expected, the "true" debris trajectory of the reference scenario (red solid line in the top plot of Figure 2) is contained in all footprint estimates.

The effectiveness of the proposed approach is shown by generating a suitable number $N_{v}$ of validation trajectories for the debris fragment and by counting how many of them actually belong to the computed footprints. $N_{v}$ is computed according to $N_{v}=\left\lceil\frac{\log (2 / \eta)}{2 \alpha^{2}}\right\rceil$, so as to ensure an $\alpha$ level of accuracy with a confidence $1-\eta$, where $\alpha$ and $\eta$ are chosen to match the parameters in Algorithm 1. The $N_{v}$ validation trajectories are generated as follows. The initial state is set equal to the "true" debris state vector right before it enters the airspace region where the stochastic wind field is present. The wind field of each realization is initialized with the true value of the wind field at the same time instant. In each trajectory the wind field evolves according to (13), and the debris object evolves according to (5) subject to the corresponding wind realization and to a realization of the Gaussian acceleration process $\boldsymbol{\xi}(t)$ with zero mean and covariance matrix $\Xi$. As pointed out in Figure 2, all the validation trajectories (reported as black dots in the top plot) belong to the computed footprints at the corresponding time instant.

\section{CONCLUSIONS}

We presented a novel simulation-based approach to solve the problem of estimating the region of the airspace posed at risk by a reentering space debris. The proposed method was shown to outperform the competing alternative covariance propagation method and to be of more general applicability. We also demonstrated how the obtained footprint estimate can be significantly enhanced if measurements of the debris position after the breakup instant as well as of the aircraft positions during normal operations prior to breakup were available. Results show that randomized techniques can be a quite effective means for addressing interesting and challenging problems involving nonlinear complex dynamics. An interesting extension of this work is footprint estimation for a cloud of debris fragments generated in the breakup event.

\section{REFERENCES}

[1] C. Pardini and L. Anselmo, "Reentry predictions for uncontrolled satellites: results and challenges," in 6th IAASS Conference - Safety is Not an Option, Montreal, Canada, 2013. 
[2] R. Patera, "Risk to commercial aircraft from reentering space debris," in AAIA Atmospheric Flight Mechanics Conference, 2008.

[3] W. Ailor and P. Wilde, "Requirements for warning aircraft of reentering debris," International Association for the Advancement of Space Safety, Tech. Rep., 2008.

[4] A. Falsone, F. Noce, and M. Prandini, "A randomized approach to space debris footprint characterization," in IFAC World Congress 2014, Cape Town, South Africa, August 2014.

[5] L. Anselmo and C. Pardini, "Satellite reentry predictions for the Italian civil protection authorities," in 63rd International Astronautical Congress. Naples, Italy: International Astronautical Federation (IAF), 2013.

[6] M. Weaver, R. Baker, and M. Frank, "Probabilistic estimation of reentry debris area," ESA SP-473, vol. 2, pp. 515-520, 2011.

[7] M. Frank, M. Weaver, and R. Baker, "A probabilistic paradigm for spacecraft random reentry disassembly," Reliability Engineering and System Safety, vol. 90, no. 2-3, pp. 148-161, 2005.

[8] C. Pardini and L. Anselmo, "Computational methods for reentry trajectories and risk assessment," Advances in Space Research, vol. 35, no. 7, pp. 1343-1352, 2005.

[9] A. Saunders, H. Lewis, and G. Swinerd, "A new tool for satellite reentry predictions," in 5th European Conference on Space Debris. Darmstadt, Germany: European Space Agency, 2009.

[10] "Flight safety analysis," Federal Aviation Administration (FAA), Washington, DC, USA, Tech. Rep. 1.0, 2011.

[11] S. Julier and J. Uhlmann, "Unscented filtering and nonlinear estimation," in Proceedings of the IEEE, vol. 92, no. 3, 2004.

[12] I. Lymperopoulos, "Sequential Monte Carlo metohds in air traffic management," PhD Thesis, ETH, Zurich - Department of Information Technology and Electrical Engineering, 2010.

[13] J. Hu, M. Prandini, and S. Sastry, "Aircraft conflict prediction in the presence of a spatially correlated wind field," IEEE Transactions on Intelligent Transportation Systems, vol. 6, no. 3, pp. 326-340, 2005.

[14] M. Prandini, L. Piroddi, S. Puechmorel, and S. L. Brazdilova, "Toward air traffic complexity assessment in new generation air traffic management systems," IEEE Transactions on Intelligent Transportation Systems, vol. 12, no. 3, pp. 809-818, 2011.

[15] M. Reyhanoglu and J. Alvarado, "Estimation of debris dispersion due to a space vehicle breakup during reentry," Acta Astronauta, vol. 86, pp. 211-218, 2013.

[16] A. Kurzhanski and P. Varaiya, "Ellipsoidal techniques for reachability under state constraints," SIAM Journal on Control and Optimization, vol. 45, no. 4, pp. 1369-1394, 2006.

[17] A. Kurzhanskiy and P. Varaiya, "Ellipsoidal techniques for reachability analysis of discrete-time linear systems," IEEE Transactions on Automatic Control, vol. 52, no. 1, pp. 26-38, Jan 2007.

[18] M. Althoff, O. Stursberg, and M. Buss, "Computing reachable sets of hybrid systems using a combination of zonotopes and polytopes," Nonlinear Analysis: Hybrid Systems, vol. 4, no. 2, pp. 233-249, 2010.

[19] C. L. Guernic and A. Girard, "Reachability analysis of linear systems using support functions," Nonlinear Analysis: Hybrid Systems, vol. 4, no. 2, pp. 250-262, 2010.

[20] G. Frehse, R. Kateja, and C. L. Guernic, "Flowpipe approximation and clustering in space-time," in Hybrid Systems: Computation and Control, 2013, pp. 203-212.

[21] M. Althoff, "Reachability analysis of nonlinear systems using conservative polynomialization and non-convex sets," in Hybrid Systems. Computation and Control, 2013, pp. 173-182.

[22] M. Prandini and J. Hu, "A stochastic approximation method for reachability computations," in Stochastic hybrid systems: theory and safety applications, ser. Lecture Notes in Control and Information Sciences 337, H. Blom and J. Lygeros, Eds. Springer Verlag, 2006, pp. 107139.

[23] — "Stochastic reachability: Theory and numerical approximation," in Stochastic hybrid systems, ser. Automation and Control Engineering Series 24, C. Cassandras and J. Lygeros, Eds. Taylor \& Francis Group/CRC Press, 2006, pp. 107-138.

[24] A. Abate, M. Prandini, J. Lygeros, and S. Sastry, "Probabilistic reachability and safety for controlled discrete time stochastic hybrid systems," Automatica, vol. 44, no. 11, pp. 2724-2734, 2008.

[25] S. Boyd, Convex Optimization. Cambridge University Press, 2004

[26] A. Prèkopa, Stochastic Programming. Boston, MA: Kluwer, 1995.

[27] — , "Probabilistic programming," in Stochastic Programming, ser. handbooks in operations research and management science, A. Ruszczyǹski and A. Shapiro, Eds., vol. 10. London, UK: Elsevier, 2003.
[28] M. Campi, S. Garatti, and M. Prandini, "The scenario approach for systems and control design," Annual Reviews in Control, vol. 33, no. 2, p. $149157,2009$.

[29] M. Campi and S. Garatti, "A sampling-and-discarding approach to chance-constrained optimization: feasibility and optimality," Journal of Optimization Theory and Applications, vol. 148, no. 2, pp. 257-280, 2011.

[30] M. Todd and E. Yildirim, "On Khachiyan's algorithm for the computation of minimum-volume enclosing ellipsoids," Discrete Applied Mathematics, vol. 155, pp. 1731-1744, 2007.

[31] P. Sun and R. Freund, "Computation of minimum-volume covering ellipsoids," Operations Research, vol. 52, no. 5, pp. 690-706, 2004.

[32] T. Alamo, R. Tempo, and A. Luque, "On the sample complexity of randomized approaches to the analysis and design under uncertainty," in Proceedings of the American Control Conference, Baltimore, MD, USA, 2010, pp. 4671-4676.

[33] A. Hedin, E. Fleming, A. Manson, F. Schmidlin, S. Avery, R. Clark, S. Franke, G. Fraser, F. V. T. Tsuda, and R. Vincent, "Empirical wind model for the upper, middle and lower atmosphere," Journal of Atmospheric and Terrestrial Physics, vol. 58, no. 13, pp. 1421-1447, 1996.

[34] U.S. Standard Atmosphere. U.S. Government Printing Office, Washington, D.C., 1976.

[35] S. Julier, "The Scaled Unscented Transformation," in Proceedings of the American Control Conference, 2002.

[36] I. Lymperopoulos and J. Lygeros, "Adaptive aircraft trajectory prediction using particle filters," in AIAA Guidance, Navigation and Control Conference and Exhibit. American Institute of Aeronautics and Astronautics, 2008.

[37] — - "Sequential Monte Carlo methods for multi-aircraft trajectory prediction in air traffic management," International Journal of Adaptive Control and Signal Processing, vol. 24, pp. 830-849, 2010.

[38] J. Lygeros and M. Prandini, "Stochastic hybrid systems: a powerful framework for complex, large scale applications," European Journal of Control, vol. 16, no. 6, pp. 583-594, 2010.

[39] User Manual for the Base of Aircraft Data, 3rd ed., Eurocontrol, 2012.

[40] R. Cole, C. Richard, S. Kim, and D. Bailey, "An assessment of the 60 $\mathrm{km}$ Rapid Update Cycle (RUC) with near real-time aircraft reports," National Aeronautics and Space Administration (NASA), Tech. Rep. July 1998

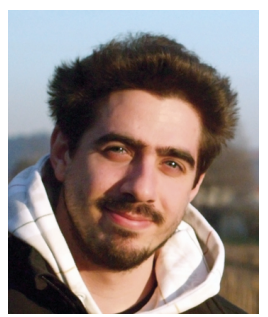

Alessandro Falsone was born in Milan (Italy), in 1989. He obtained the Bachelor of Science in 2011 and the Master of Science cum laude in 2013, both in Automation and Control Engineering. From November 2013 to October 2014 he worked as a research assistant at the Dipartimento di Elettronica, Informazione e Bioingegneria at Politecnico di Milano. Since November 2014 he is a Ph.D. student in the System and Control division of the same department. His current research interests include distributed optimization and control, optimal control of stochastic hybrid systems, randomized algorithms, and nonlinear model identification

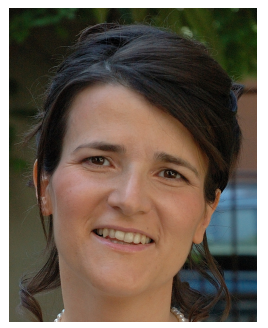

Maria Prandini received her laurea degree in Electrical Engineering (summa cum laude) from Politecnico di Milano (1994) and her Ph.D. degree in Information Technology from Universitá degli Studi di Brescia, Italy (1998). From 1998 to 2000 she was a postdoctoral researcher at the Department of Electrical Engineering and Computer Sciences, University of California at Berkeley. She also held visiting positions at Delft University of Technology (1998), Cambridge University (2000), University of California at Berkeley (2005), and Swiss Federal Institute of Technology Zurich (2006). In 2002, she started as an assistant professor in systems and control at Politecnico di Milano, where she is currently an associate professor. Her research interests include stochastic hybrid systems, randomized algorithms, constrained control, system abstraction and verification, nonlinear identification, distributed optimization, and the application of control theory to air traffic management and energy systems. She serves on the editorial board of Cyber Physical Systems, and previously of European Journal of Control, IEEE Transactions on Automatic Control, IEEE Transactions on Control Systems Technology and Nonlinear Analysis: Hybrid Systems. From 2013 to 2105, she has been editor for Electronic Publications of the IEEE CSS. She is member of the IEEE CSS Board of Governors, and since January 2016 she is CSS Vice-President for Conference Activities. 